The Astrophysical Journal, 681:1318-1326, 2008 July 10

(C) 2008. The American Astronomical Society. All rights reserved. Printed in U.S.A.

\title{
NEW THEORETICAL RESULTS CONCERNING THE INTERSTELLAR ABUNDANCE OF MOLECULAR OXYGEN
}

\author{
DonghuI Quan \\ Chemical Physics Program, The Ohio State University, Columbus, OH 43210 \\ ERIC HerbST \\ Departments of Physics, Astronomy, and Chemistry, The Ohio State University, Columbus, OH 43210 \\ T. J. MiLlaR \\ Astrophysics Research Centre, School of Mathematics and Physics, Queen's University Belfast, Belfast BT7 1NN, Northern Ireland \\ George E. Hassel \\ Department of Physics, The Ohio State University, Columbus, OH 43210 \\ SHI Ying Lin and Hua GuO \\ Department of Chemistry and Chemical Biology, University of New Mexico, Albuquerque, NM 87131 \\ Pascal Honvault \\ Institut UTINAM, UMR CNRS 6213, University of Franche-Comté, Campus de la Bouloie, \\ UFR Sciences et Techniques, 25030 Besançon Cedex, France \\ AND \\ DAIQIAN Xie \\ Institute of Theoretical and Computational Chemistry, Key Laboratory of Mesoscopic Chemistry, \\ School of Chemistry and Chemical Engineering, Nanjing University, Nanjing 210093, China \\ Received 2007 August 21; accepted 2008 March 4
}

\begin{abstract}
The low abundance of molecular oxygen in cold cores of interstellar clouds poses a continuing problem to modelers of the chemistry of these regions. In chemical models $\mathrm{O}_{2}$ is formed principally by the reaction between $\mathrm{O}$ and $\mathrm{OH}$, which has been studied experimentally down to $39 \mathrm{~K}$. It remains possible that the rate coefficient of this reaction at $10 \mathrm{~K}$ is considerably less than its measured value at $39 \mathrm{~K}$, which might inhibit the production of $\mathrm{O}_{2}$ and possibly bring theory and observation closer together over a wider range of times. Two theoretical determinations of the rate coefficient for the $\mathrm{O}+\mathrm{OH}$ reaction at temperatures down to $10 \mathrm{~K}$ have been undertaken recently; both results show that the rate coefficient is indeed lower at $10 \mathrm{~K}$ than at $39 \mathrm{~K}$, although they differ in the magnitude of the decrease. Here we show, using gas-phase models, how the calculated interstellar $\mathrm{O}_{2}$ abundance in cold cores is affected by a decrease in the rate coefficient. We also consider its effect on other species. Our major finding is that for standard O-rich abundances, the calculated abundance of $\mathrm{O}_{2}$ in cold cores is sufficiently low to explain observations only at early times regardless of the value of $k_{1}$ in the range investigated here. For C-rich abundances, on the other hand, late-time solutions can also be possible.
\end{abstract}

Subject headings: astrochemistry — ISM: abundances — ISM: clouds — ISM: molecules

\section{INTRODUCTION}

The lack of detection of $\mathrm{O}_{2}$ and $\mathrm{H}_{2} \mathrm{O}$ in the gas phase of cold interstellar cores such as TMC-1 and L134N is inconsistent with steady state models of the gas-phase chemistry (Bergin et al. 2000). A large number of solutions to this discrepancy have been suggested, as discussed in Larsson et al. (2007). Perhaps the simplest is the argument that the clouds are young; pseudo-time-dependent gas-phase models of the chemistry of the large number of species seen in TMC-1 and L134N work best at early times around $10^{5} \mathrm{yr}$ (Wakelam et al. 2006). In the original calculations by Bergin et al. (2000) the $\mathrm{O}_{2}$ abundance is still rather low at this time, although the $\mathrm{H}_{2} \mathrm{O}$ abundance is already too large to explain observations. With our most recent gas-phase model calculations, however, which include a criterion for "agreement" between observation and model abundances based on the overlap of Gaussian error functions, the abundance of water is sufficiently low over a wide range of times to explain its lack of detectability in most cold sources, although the severe upper limits in B68 and $\rho$ Oph D may still present problems (see Bergin \& Snell 2002). Even though the $\mathrm{O}_{2}$ problem remains for times after $10^{5} \mathrm{yr}$ ( Wakelam et al. 2006), agreement at later times can be achieved in gas-phase models by changing the standard elemental abundances used to carbon-rich ones, although care must be taken to preserve agreement for other species as well (Bergin et al. 2000). Indeed, such models replicate the complex organic chemistry seen in TMC-1 best (Wakelam et al. 2006). Other solutions to the $\mathrm{O}_{2} / \mathrm{H}_{2} \mathrm{O}$ problems involve shocks, turbulence, bistability, and grain chemistry in some manner (Bergin et al. 2000; Charnley et al. 2001; Spaans \& van Dishoeck 2001; Viti et al. 2001; Willacy et al. 2002; Roberts $\&$ Herbst 2002). In the most recent gas-grain model study of cold cores, Garrod et al. (2007) show that small abundances of $\mathrm{O}_{2}$ and $\mathrm{H}_{2} \mathrm{O}$ can be achieved over a significant range of times even if standard O-rich abundances are utilized.

The case of $\mathrm{O}_{2}$ has been complicated by our lack of knowledge of the rate coefficient at the standard cold core temperature of $10 \mathrm{~K}$ for its major formation reaction:

$$
\mathrm{O}+\mathrm{OH} \longrightarrow \mathrm{O}_{2}+\mathrm{H}
$$


Until recently, the rate coefficient $k_{1}$ used in the OSU network ${ }^{1}$ was estimated from higher temperature measurements to be given by the expression $7.5 \times 10^{-11}(T / 300)^{-0.25} \mathrm{~cm}^{3} \mathrm{~s}^{-1}$, leading to a value at $10 \mathrm{~K}$ of $1.76 \times 10^{-10} \mathrm{~cm}^{3} \mathrm{~s}^{-1}$. The value in the RATE99 network (Le Teuff et al. 2000) was $1.77 \times 10^{-11} \exp (178 / T) \mathrm{cm}^{3} \mathrm{~s}^{-1}$, but pertains only to temperatures above $150 \mathrm{~K}$. Recent experiments in the temperature range 39-142 K with the CRESU (Cinétique de Réaction en Ecoulement Supersonique Uniforme) technique (Carty et al. 2006) show that the rate coefficient is a constant $3.5 \times 10^{-11} \mathrm{~cm}^{3} \mathrm{~s}^{-1}$. The more recent RATE06 database ${ }^{2}$ contains this value for temperatures under $142 \mathrm{~K}$. Although the temperature independence of the CRESU result is valid, the value itself may be somewhat low according to measurements of Smith \& Stewart (1994) and Robertson \& Smith (2006). Changes in the calculated abundance of $\mathrm{O}_{2}$ as a result of the lowering of the rate coefficient $k_{1}$ to the $39 \mathrm{~K}$ experimental value have been discussed by T. J. Millar at a talk at the annual network meeting of "The Molecular Universe" in Perugia, Italy, 2007 March.

Recently, Xu et al. (2007b) have undertaken quantum mechanical calculations of the reaction rate coefficient at low temperature by a variety of approaches (see below). With the $J$-shifting approximation and the neglect of nonadiabatic coupling, they obtained a rate coefficient that decreases as the temperature drops from 100 to $10 \mathrm{~K}$. At $40 \mathrm{~K}$, the calculated rate coefficient is somewhat under $2 \times 10^{-11} \mathrm{~cm}^{3} \mathrm{~s}^{-1}$, or about $\frac{1}{2}$ of the experimental value. By $10 \mathrm{~K}$, the computed rate coefficient has fallen to a value of $5.4 \times 10^{-13} \mathrm{~cm}^{3} \mathrm{~s}^{-1}$, significantly under the $39 \mathrm{~K}$ experimental value. More recent calculations (Lin et al. 2008a), in which the $J$-shifting approximation has been removed, show that the drop in rate coefficient as the temperature decreases is nowhere near as great, with the value at $10 \mathrm{~K}$ only $7.84 \times 10^{-12} \mathrm{~cm}^{3} \mathrm{~s}^{-1}$, or about $1 / 4.5$ of the experimental value at $39 \mathrm{~K}$. A decrease in rate coefficient at low temperatures had previously been discussed by Harding et al. (2000).

Here we report pseudo-time-dependent calculations of the gasphase chemistry of homogeneous cold interstellar cloud cores to determine the effect of the rate coefficient for the reaction between $\mathrm{O}+\mathrm{OH}$ on the abundances of $\mathrm{O}_{2}, \mathrm{OH}$, and other species. We consider $\mathrm{OH}$ because a lowering of $k_{1}$ can result in a greater abundance of this radical if reaction (1) is an important depletion mechanism. Moreover, the abundances of many other species, mainly oxygen containing, are affected by varying the rate coefficient of reaction (1), even though they are only indirectly involved. We compare our results with observations in TMC-1 and L134N. For $\mathrm{O}_{2}$ we utilize the upper limits to the fractional abundance determined via the Odin satellite. These limits with respect to $\mathrm{H}_{2}$ are $1.7 \times 10^{-7}$ in L134N and $7.7 \times 10^{-8}$ in TMC- 1 (Pagani et al. 2003). For the abundance of $\mathrm{OH}$ in TMC-1 we use the fractional abundance, $2 \times 10^{-7}$, determined by Harju et al. (2000) from mapping TMC-1 at $1665 \mathrm{MHz}$ using the Effelsberg $100 \mathrm{~m}$ telescope. Although the beam size is large, $7.8^{\prime}$, at this low frequency, it corresponds well to the $10^{\prime}$ resolution of the Odin satellite at the $\mathrm{O}_{2}$ frequency of $118.75 \mathrm{GHz}$. We adopt a value of $7.5 \times 10^{-8}$ for L134N (Smith et al. 2004; Ohishi et al. 1992).

The remainder of the paper is organized as follows. In $\S 2$ we summarize our quantum chemical calculations. Section 3 contains a discussion of our chemical model and results for $\mathrm{O}_{2}, \mathrm{OH}$, and other species with a variety of elemental abundances. It also contains a discussion of the $\mathrm{O}_{2}$ problem in warm sources. The paper concludes with a general discussion and a speculation on

\footnotetext{
1 See http://www.physics.ohio-state.edu/ eric/research.html.

2 See http://www.udfa.net/.
}

what might be learned from either an actual detection or reduction of the upper limit of molecular oxygen in cold cores.

\section{QUANTUM MECHANICAL CALCULATIONS}

The quantum mechanical calculation of the rate coefficient was carried out on a newly developed potential energy surface for $\mathrm{HO}_{2}\left(X^{2} A^{\prime \prime}\right.$; Xu et al. 2005). Two dynamical models were used. The first computed the rate coefficient from the total angular momentum $J=0$ result using the $J$-shifting approximation (Xu et al. 2007b). The second is dynamically exact because it includes all Coriolis couplings and computes all partial wave contributions. Specifically, for each $J$ up to 150 , a wave packet corresponding to $\mathrm{OH}(j=0, v=0)$ was launched from the $\mathrm{O}+\mathrm{OH}$ asymptotic channel and propagated with a Chebyshev propagator. The total reaction probability was then calculated in the $\mathrm{H}+\mathrm{O}_{2}$ asymptote using a flux method. These total reaction probabilities were then used to obtain the integral cross section by a partial wave sum. The rate coefficient was finally obtained by Boltzmann averaging over a range of collision energies. This wave packet approach was discussed in detail by Lin \& Guo (2004).

It is known that there are 36 fine-structure states in the $\mathrm{OH}\left({ }^{2} \Pi\right)+\mathrm{O}\left({ }^{3} P\right)$ asymptote due to the radical nature of the two reactants (Graff \& Wagner 1990). However, an earlier analysis of the nonadiabaticity in this system suggested a negligible impact on the reactivity. As a result, our calculations neglected the nonadiabatic coupling in this channel. Instead, the temperaturedependent electronic partition function of Graff \& Wagner (1990)

$Q_{\mathrm{el}}=[5+3 \exp (-228 / T)+\exp (-326 / T)][1+\exp (-205 / T)]$

was used.

The work of Davidsson \& Stenholm (1990) addressed the same problem as discussed here. However, the reliability of their results is in serious question because the dynamical models used in their calculations are inaccurate, and the potential energy surface has also been shown to be problematic. In fact, Guo and coworkers have recently published a series of papers that showed the following: (1) the potential energy surface used for the $\mathrm{HO}_{2}$ system by Davidsson \& Stenholm (1990) has significant flaws that lead to large errors in the $\mathrm{HO}_{2}$ vibrational spectrum and in reaction dynamics (Xu et al. 2005, 2007a, 2007b; Lin et al. 2006a, $2006 \mathrm{~b}$ ), and (2) the $\mathrm{O}+\mathrm{OH}$ reaction is nonstatistical (Miller 1986; Lin et al. 2008a, 2008b). Thus, the capture model used by Davidsson \& Stenholm (1990) is not expected to yield reliable rate coefficients.

\section{CHEMICAL MODEL AND RESULTS}

The osu.01.2007 gas-phase chemical network has been utilized with a hydrogen nuclear density $n_{\mathrm{H}}=2 \times 10^{4} \mathrm{~cm}^{-3}$, a visual extinction $A_{V}=10$, a cosmic-ray ionization parameter $\zeta=1.3 \times$ $10^{-17} \mathrm{~s}^{-1}$, and a temperature $T=10 \mathrm{~K}$, the standard parameters for cold cores. Analogous calculations using the RATE06 data set lead in the main to very similar results, especially if the dipole option is chosen. It should be mentioned that recent observations, especially of nitrogen-bearing species, reveal that L134N does have a dense central condensation and is best thought of as a prestellar core (Pagani et al. 2007). Two sets of so-called lowmetal elemental abundances were utilized. For L134N we used the standard O-rich abundances, whereas for TMC-1 we used C-rich abundances, which have proven most useful in improving agreement with observation for the more than 50 detected molecules in this source (Wakelam et al. 2006). The level of 
TABLE 1

Initial Nonzero Fractional Abundances

\begin{tabular}{|c|c|c|}
\hline Species & L134N & TMC-1 \\
\hline He..... & $6.00 \times 10^{-2}$ & $6.00 \times 10^{-2}$ \\
\hline$\ldots \ldots \ldots$ & $2.14 \times 10^{-5}$ & $2.14 \times 10^{-5}$ \\
\hline $\mathrm{O} \ldots \ldots \ldots$ & $1.76 \times 10^{-4}$ & $6.10 \times 10^{-5}$ \\
\hline $\mathrm{H}_{2} \ldots \ldots \ldots \ldots \ldots \ldots \ldots \ldots \ldots \ldots \ldots \ldots \ldots \ldots \ldots \ldots \ldots$ & $5.00 \times 10^{-1}$ & $5.00 \times 10^{-1}$ \\
\hline $\mathrm{C}^{+} \ldots \ldots$ & $7.30 \times 10^{-5}$ & $7.30 \times 10^{-5}$ \\
\hline $\mathrm{S}^{+} \ldots \ldots$ & $8.00 \times 10^{-8}$ & $8.00 \times 10^{-8}$ \\
\hline $\mathrm{Si}^{+} \ldots$ & $8.00 \times 10^{-9}$ & $8.00 \times 10^{-9}$ \\
\hline $\mathrm{Fe}^{+} \ldots$ & $3.00 \times 10^{-9}$ & $3.00 \times 10^{-9}$ \\
\hline $\mathrm{Na}^{+} \ldots$ & $2.00 \times 10^{-9}$ & $2.00 \times 10^{-9}$ \\
\hline $\mathrm{Mg}^{+} \ldots$ & $7.00 \times 10^{-9}$ & $7.00 \times 10^{-9}$ \\
\hline $\mathrm{P}^{+} \ldots \ldots \ldots$ & $3.00 \times 10^{-9}$ & $3.00 \times 10^{-9}$ \\
\hline $\mathrm{Cl}^{+}$. & $4.00 \times 10^{-9}$ & $4.00 \times 10^{-9}$ \\
\hline$\ldots \ldots \ldots .$. & $6.69 \times 10^{-9}$ & $6.69 \times 10^{-9}$ \\
\hline
\end{tabular}

agreement at early time can be seen in Wakelam et al. (2006), with the actual time of best agreement $\left(\leq 10^{5} \mathrm{yr}\right)$ dependent on the algorithm used. The two sets of elemental abundances are shown in Table 1 in the form of the initial chemical species with nonzero fractional abundances with respect to total hydrogen nuclei. To allow for a possible high abundance of sulfur in the gas phase, we also used a sulfur abundance 2 orders of magnitude higher than the "low-metal" one, while abundances of other elements remained the same. The results are discussed separately in $\S 3.3$ below. In our calculations, we used the four values for the rate coefficient $k_{1}$ of reaction (1) shown and explained in Table 2.

In discussing the surprisingly complex chemistry of $\mathrm{O}_{2}$ and its precursor $\mathrm{OH}$, we will use two simplifying terms for reaction (1): dominant and rate limiting. The term "dominant" here means that the reaction is the major route for the formation of $\mathrm{O}_{2}$, while the term "rate limiting" means that the reaction is the major process for the destruction of $\mathrm{OH}$. If the reaction is both dominant and rate limiting, then a change in its rate coefficient will not affect the abundance of $\mathrm{O}_{2}$ and will strongly affect the abundance of $\mathrm{OH}$. This can be shown mathematically under time-dependent or steady state conditions. For simplicity, we choose the latter. Assume that $\mathrm{OH}$ is depleted by reaction (1), as well as by reactions with ions and other neutrals. Then, at steady state,

$$
[\mathrm{OH}]=f /\left(k_{1}[\mathrm{O}]+k_{\mathrm{i}}\left[\mathrm{I}^{+}\right]+k_{\mathrm{n}}[\mathrm{N}]\right),
$$

where $f$ is the $\mathrm{OH}$ formation rate, $k_{\mathrm{i}}$ and $k_{\mathrm{n}}$ are the rate coefficients for reaction of $\mathrm{OH}$ with ions $\mathrm{I}^{+}$and neutrals $\mathrm{N}$ other than $\mathrm{O}$, respectively, and the brackets refer to concentration or fractional abundance. If reaction (1) is rate limiting, the abundance of $\mathrm{OH}$ reduces to the expression

$$
[\mathrm{OH}]=f /\left(k_{1}[\mathrm{O}]\right) .
$$

Thus, an increase in the value of $k_{1}$, as long as it does not have a strong affect on the abundance of atomic oxygen, will reduce the calculated abundance of $\mathrm{OH}$.

Now consider the steady state abundance of $\mathrm{O}_{2}$, which is given by the expression

$$
\left[\mathrm{O}_{2}\right]=\frac{k_{1}[\mathrm{O}][\mathrm{OH}]+f^{\prime}}{k_{\mathrm{c}}[\mathrm{C}]+k_{\mathrm{i}}\left[\mathrm{I}^{+}\right]}
$$

where $f^{\prime}$ represents alternative formation routes to $\mathrm{O}_{2}$ from reaction (1) and $k_{\mathrm{c}}$ is the rate coefficient for the destruction reaction for $\mathrm{O}_{2}$ with atomic carbon. If, in addition to rate limiting for $\mathrm{OH}$,

\begin{tabular}{|c|c|c|}
\hline $\begin{array}{c}k_{1} \\
\left(\mathrm{~cm}^{3} \mathrm{~s}^{-1}\right)\end{array}$ & Remarks & Reference \\
\hline $1.76 \times 10^{-10}$ & osu.1.2007 & Smith et al. (2004) \\
\hline $3.5 \times 10^{-11}$ & Experimental $(39-142 \mathrm{~K})$ & Carty et al. (2006) \\
\hline $7.84 \times 10^{-12} \ldots \ldots \ldots$ & Theoretical without $J$-shifting & This work \\
\hline $5.4 \times 10^{-13} \ldots \ldots \ldots$ & Theoretical with $J$-shifting & Xu et al. (2007b) \\
\hline
\end{tabular}

TABLE 2

Values Used for Rate Coefficient $k_{1}$ at $10 \mathrm{~K}$

reaction (1) is dominant for $\mathrm{O}_{2}$ formation, then the abundance of $\mathrm{O}_{2}$ is given by the expression

$$
\left[\mathrm{O}_{2}\right]=\frac{k_{1}[\mathrm{O}][\mathrm{OH}]}{k_{\mathrm{c}}[\mathrm{C}]+k_{\mathrm{i}}\left[\mathrm{I}^{+}\right]} \approx \frac{f}{k_{\mathrm{c}}[\mathrm{C}]+k_{\mathrm{i}}\left[\mathrm{I}^{+}\right]},
$$

which has been derived by substitution of equation (4) and the inequality $f>f^{\prime}$. The expression is seen to be independent of $k_{1}$. But, if reaction (1) is not the dominant process for the formation of $\mathrm{O}_{2}$, then a change in its rate coefficient will also not affect the $\mathrm{O}_{2}$ abundance directly, since the numerator of reaction (5) will be $\approx f^{\prime}$. Also, if reaction (1) is not rate limiting, then it will not affect the abundance of $\mathrm{OH}$ strongly and so will affect the abundance of $\mathrm{O}_{2}$ if it is dominant. Finally, if reaction (1) is neither rate limiting nor dominant, it affects neither species. It must be remembered, however, that in a complex network of reactions, the terms "rate limiting" and "dominant" often have to be qualified. For example, reaction (1) can be rate limiting for $\mathrm{OH}$ only within a limited range of $k_{1}$ values, because other reactions can become more important as $k_{1}$ decreases.

\section{1. $\mathrm{OH}$ and $\mathrm{O}_{2}$}

Our results for the fractional abundances of $\mathrm{OH}$ and $\mathrm{O}_{2}$ with respect to $\mathrm{H}_{2}$ in $\mathrm{L} 134 \mathrm{~N}$ (O-rich abundances) as functions of time are shown in Figures 1 and 2, respectively. Let us first look at the

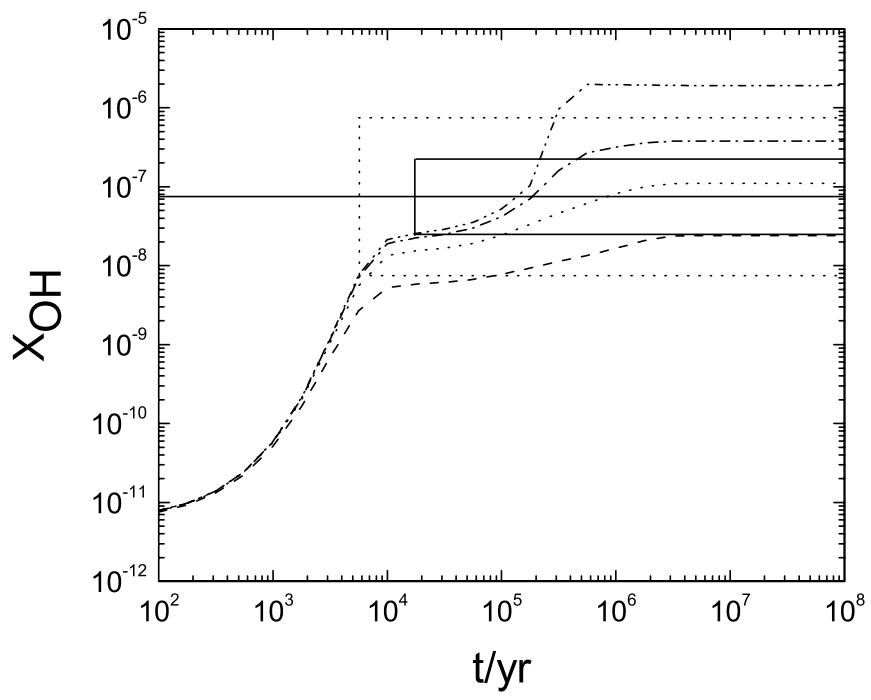

FIG. 1.-Fractional abundance of $\mathrm{OH}$ with respect to $\mathrm{H}_{2}$ plotted as a function of time for four different values of the rate coefficient $k_{1}$. O-rich abundances relevant to $\mathrm{L} 134 \mathrm{~N}$ are used. The observed fractional abundance is depicted as a solid horizontal line. Dashed line: $k_{1}=1.76 \times 10^{-10} \mathrm{~cm}^{3} \mathrm{~s}^{-1}$; dotted line: $k_{1}=$ $3.5 \times 10^{-11} \mathrm{~cm}^{3} \mathrm{~s}^{-1}$; dash-dotted line: $k_{1}=7.84 \times 10^{-12} \mathrm{~cm}^{3} \mathrm{~s}^{-1}$; dot-dotdashed line: $k_{1}=5.4 \times 10^{-13} \mathrm{~cm}^{3} \mathrm{~s}^{-1}$. The boxes delineate the regions where agreement between observation and theory is within a factor of 3 (solid line) and a factor of 10 (dotted line). 


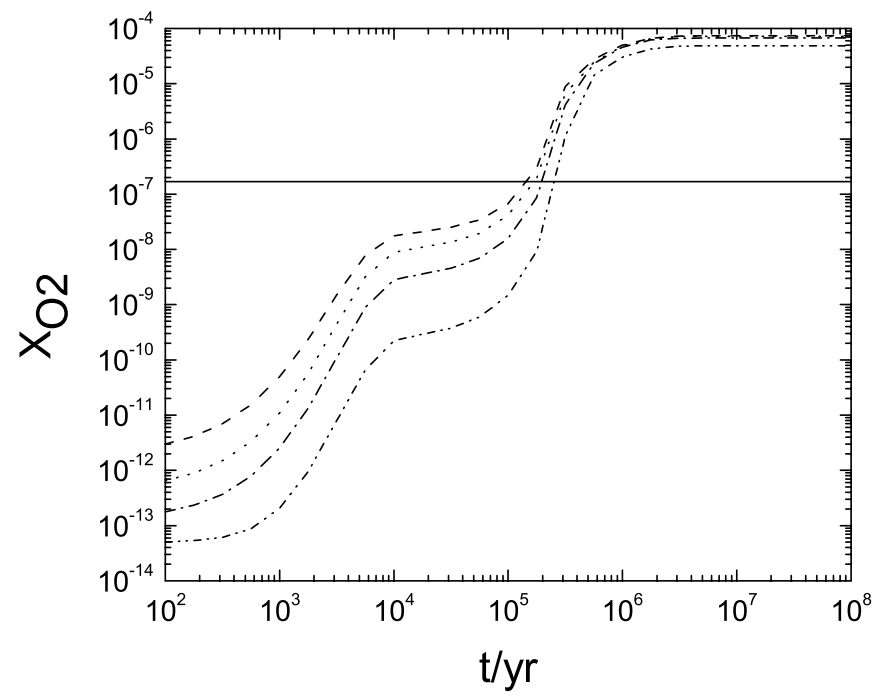

FIG. 2.-Fractional abundance of $\mathrm{O}_{2}$ with respect to $\mathrm{H}_{2}$ plotted as a function of time for four different values of the rate coefficient $k_{1}$. O-rich abundances relevant to $\mathrm{L} 134 \mathrm{~N}$ are used. The observed upper limit is depicted as a solid horizontal line. See Fig. 1 caption for $k_{1}$ values of different curves.

results for $\mathrm{OH}$ in Figure 1. The abundance of $\mathrm{OH}$ increases with time until about $10^{6} \mathrm{yr}$. The dependence of the abundance of $\mathrm{OH}$ on the value of $k_{1}$ also increases as time increases, so that at steady state there is a 2 order of magnitude divergence between the lowest abundance, corresponding to the highest value of $k_{1}$, and the highest abundance, corresponding to the lowest value of $k_{1}$. From our discussion above, reaction (1) would appear to be rate limiting at late times near steady state. Detailed model results show, however, that the reaction is not the only important destruction process for $\mathrm{OH}$ at late times, especially for the smallest $k_{1}$, and that reactions with protonating ions also contribute. At earlier times, $\mathrm{OH}$ is depleted mainly by reaction with $\mathrm{C}^{+}$, as well as with neutral $\mathrm{C}$ and N. Figure 1 also contains two boxes showing at what times and for which values of $k_{1}$ the calculated $\mathrm{OH}$ abundance is within a factor of 3 and a factor of 10 of the observed value. As noted by the referee, the low-frequency observations of $\mathrm{OH}$, and consequent large telescopic beams, may not pertain solely to the dense portions of cold cores so that the observational uncertainty may indeed be larger than a factor of 3. On the other hand, the mapping observations of $\mathrm{OH}$ by Harju et al. (2000) indicate that the $1665 \mathrm{MHz}$ line is likely optically thin. Their analysis indicates that its distribution and line parameters are similar to those of species such as $\mathrm{CCS}, \mathrm{HC}_{3} \mathrm{~N}$, and $\mathrm{C}_{4} \mathrm{H}$, species whose line emission arises from dense gas rather than the surrounding envelope. Indeed, the $\mathrm{OH}$ column density has its maximum value close to the cyanopolyyne peak, indicating that the bulk of the $\mathrm{OH}$ emission arises from the dense core of TMC-1.

Using the factor-of-3 criterion for agreement between theory and observation for $\mathrm{OH}$ (Wakelam et al. 2006), we see from the box in Figure 1 that the best agreement for L134N (O-rich abundances) depends on the time, as well as the value of $k_{1}$. For the highest value of $k_{1}$, agreement starts only at times later than $10^{6} \mathrm{yr}$, while for the lowest value, agreement is best around $10^{5} \mathrm{yr}$ but rapidly deteriorates with increasing time. For the experimental value of the rate coefficient agreement is reasonable over the largest period of time. If we use a factor of 10 for the criterion for agreement, the constraints are less tight.

Let us now look at the results in Figure 2. We see that the $\mathrm{O}_{2}$ fractional abundance increases steadily with time until reaching a very high steady state value of almost $10^{-4}$ with respect to $\mathrm{H}_{2}$

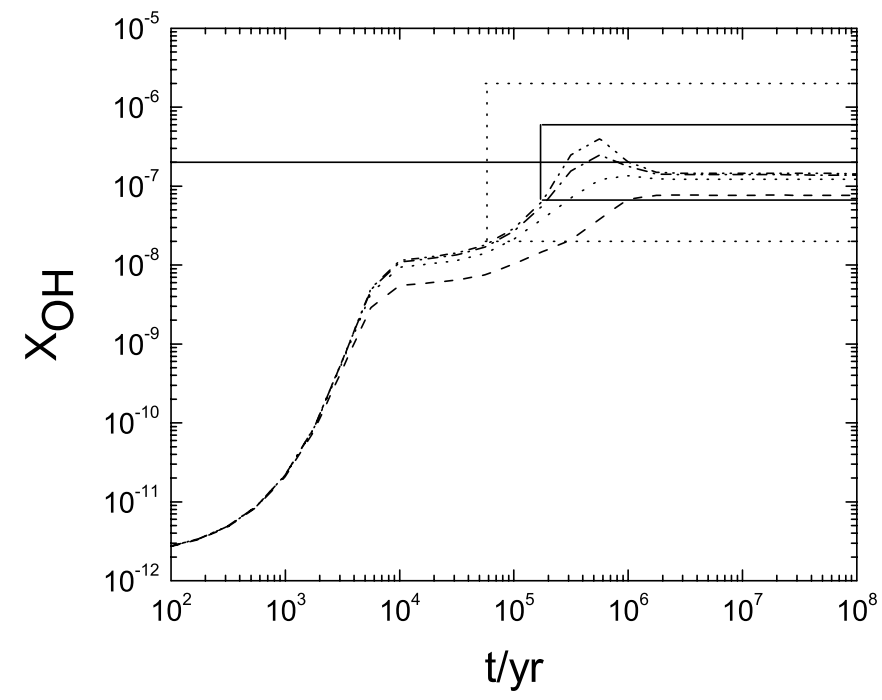

FIG. 3.- Same as Fig. 1, but C-rich abundances relevant to TMC-1 are used.

somewhat after $10^{6} \mathrm{yr}$, as shown before by Bergin et al. (2000). Before this time, the abundance of $\mathrm{O}_{2}$ depends directly on the value of $k_{1}$. For example, at a time of $10^{5} \mathrm{yr}$, the fractional abundances range from $\approx 10^{-9}$ for the lowest value of $k_{1}$ to almost $10^{-7}$ for the highest value of $k_{1}$. As the time increases slightly to $3 \times 10^{5} \mathrm{yr}$, all abundances increase strongly but the smaller abundances increase the more strongly so that the differences become much smaller although they do not disappear completely. Regarding the observed $\mathrm{O}_{2}$ upper limit, all four models reach this value at a time around $(2-3) \times 10^{5} \mathrm{yr}$. At late times, our detailed model results show that reaction (1) accounts for between $40 \%$ and $60 \%$ of the $\mathrm{O}_{2}$ production depending on the value of $k_{1}$, and so is close to dominant as well as rate limiting. Other reactions leading to $\mathrm{O}_{2}$ include $\mathrm{HOCO}^{+}+\mathrm{O}$. At earlier times $\left(\leq 10^{5} \mathrm{yr}\right)$ reaction $(1)$ is clearly dominant (calculations show that it accounts for nearly $100 \%$ of the $\mathrm{O}_{2}$ production), but it is certainly not rate limiting.

Now let us turn to the C-rich abundances, used for TMC-1. For $\mathrm{OH}$, shown in Figure 3, the separation among the $\mathrm{OH}$ abundances for differing values of $k_{1}$ is generally not large, because reaction (1) is not rate limiting, since a lower oxygen elemental abundance is used. The solid box in Figure 3, which delineates the regions of factor-of-3 agreement, shows however that there is some difference among the different values of $k_{1}$. In particular, the largest value of $k_{1}$ once again leads to agreement only at times over $10^{6} \mathrm{yr}$, while the other three rate coefficients all lead to agreement with observation for times over $(2-3) \times 10^{5} \mathrm{yr}$. Once again, a criterion of a factor of 10 for agreement with observation leads to looser constraints, especially for the largest value of $k_{1}$.

In Figure 4, the four curves for $\mathrm{O}_{2}$ show considerable dispersion at most times, indicating that reaction (1) can be dominant. Moreover, the four values of the $\mathrm{O}_{2}$ abundance increase monotonically from the beginning of the calculation until a time near $5 \times 10^{5} \mathrm{yr}$, when peak abundances are reached. The calculated abundances then decrease until a time near $2 \times 10^{6} \mathrm{yr}$, when steady state is eventually reached. The "bump" is correlated with a declivity in the abundance of atomic carbon, which is related to $\mathrm{O}_{2}$, since the reaction

$$
\mathrm{C}+\mathrm{O}_{2} \rightarrow \mathrm{CO}+\mathrm{O}
$$

is always the main destruction reaction of $\mathrm{O}_{2}$ during this time range. With the lowest of the four values of $k_{1}$ used, the calculated $\mathrm{O}_{2}$ fractional abundance essentially lies below the observed 


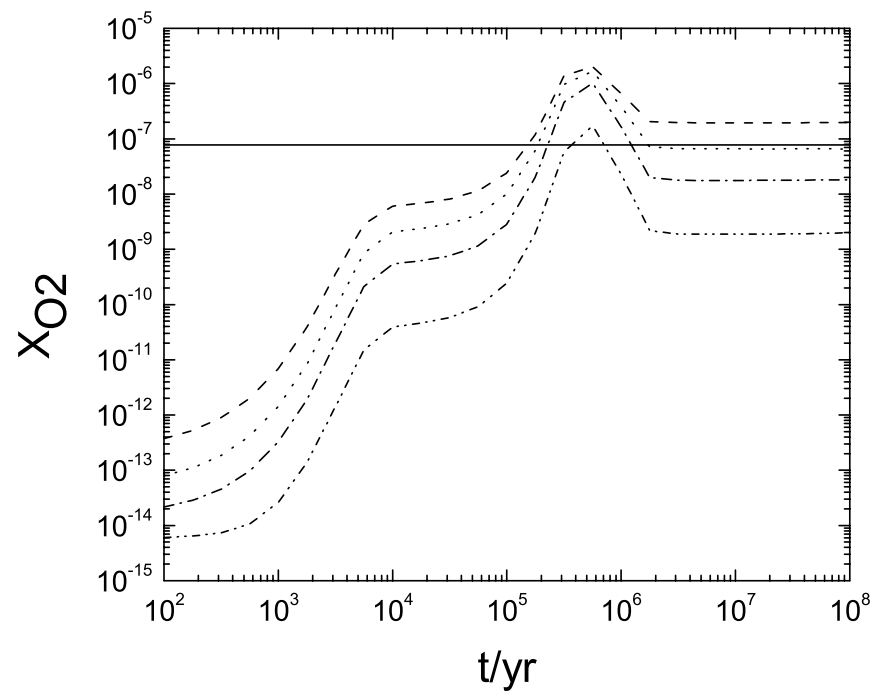

FIG. 4.- Same as Fig. 2, but C-rich abundances relevant to TMC-1 are used.

upper limit for all times. With the highest value of $k_{1}$, the calculated abundance becomes too large at a little more than $10^{5} \mathrm{yr}$. The middle two values of $k_{1}$ yield results in between these two extremes.

Summarizing the $\mathrm{O}_{2}$ problem with both sets of abundances, at the optimum early-time ages for L134N and TMC-1 determined by Wakelam et al. (2006) there is no major discrepancy with the observed upper limit of $\mathrm{O}_{2}$. Nor is there a discrepancy of note with the observed abundance of $\mathrm{OH}$ if the larger uncertainty in the observed abundance is chosen. If one insists on a later time or even steady state solution, however, $\mathrm{O}$-rich abundances produce a single very large abundance of $\mathrm{O}_{2}$ for all four values of the rate coefficient, whereas $\mathrm{C}$-rich abundances generally produce an overly large abundance of $\mathrm{O}_{2}$ only with the largest value of $k_{1}$, which probably does not pertain to $10 \mathrm{~K}$. Moreover, the large abundance does not exceed the upper limit by more than an order of magnitude. For $\mathrm{OH}$ at late times, only the smallest value of $k_{1}$ under $\mathrm{O}$-rich conditions gives trouble if the larger uncertainty is chosen.

\subsection{Other Species}

Apart from affecting the reactants and products directly involved in reaction (1), a change in the value of $k_{1}$ can influence many other species. In our calculations, we looked at the two extreme cases with the largest and the smallest $k_{1}$ values at $10 \mathrm{~K}$. Figure 5 shows the percentage of affected species for both O-rich and $\mathrm{C}$-rich cases as a function of time using the criterion of a factor of 10 change in abundance as "significant."

For the O-rich case, up to $30 \%$ of the 452 species in our model are significantly affected at one time or another. Following the example of $\mathrm{OH}$, however, these effects occur overwhelmingly at $>10^{5} \mathrm{yr}$ and so will not change the overall agreement with observation at early times. For the $\mathrm{C}$-rich elemental abundances, the effects of the change in $k_{1}$ are much smaller. Using the same criterion, only $2 \%$ of the species in our model are significantly affected. The affected species are connected to the reactants or products of reaction (1) via reaction pathways. Since there is much less atomic oxygen in the $\mathrm{C}$-rich case and the $\mathrm{OH}$ abundance is less dependent on changes in $k_{1}$, far fewer species are strongly affected.

The specific examples of $\mathrm{NO}$ and $\mathrm{SO}_{2}$ are depicted in Figure 6. The horizontal lines in these figures are the observed abundance for $\mathrm{NO}$ and the upper limit for $\mathrm{SO}_{2}$ in $\mathrm{L} 134 \mathrm{~N}$ and the observed abundances of both in TMC-1 (Ohishi et al. 1992; Smith et al.

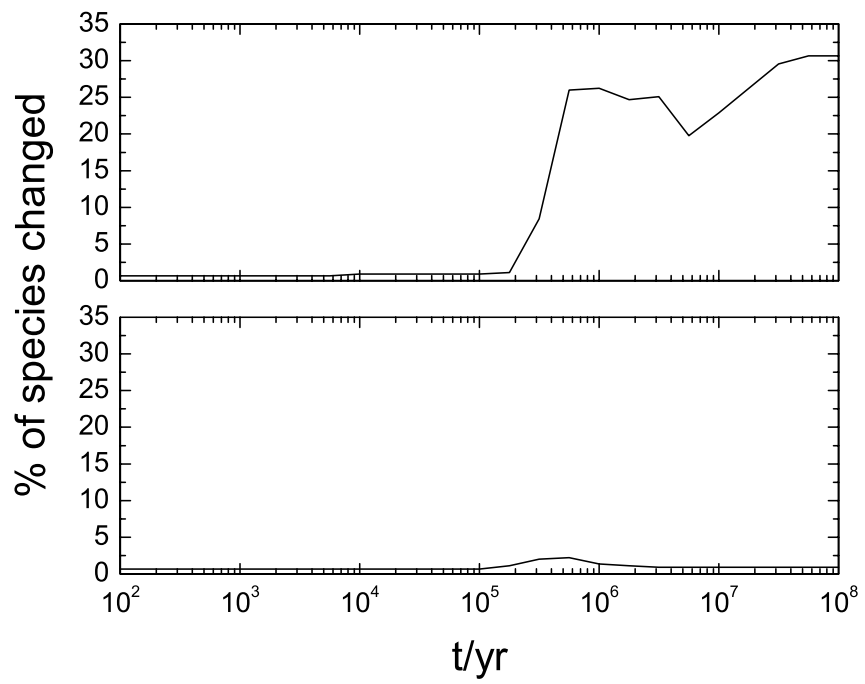

FIG. 5.- Percentage of significantly affected species plotted against time. Upper panel: O-rich case; lower panel: C-rich case.

2004). In the case of NO, the radical is produced via the reaction between $\mathrm{N}$ and $\mathrm{OH}$ and follows the $\mathrm{OH}$ abundance closely. Not only does the general increase of $\mathrm{OH}$ with time, which occurs with both sets of elemental abundances, lead to a similar increase for $\mathrm{NO}$, but for O-rich abundances, the large dispersion among the four $\mathrm{OH}$ curves at late times leads to a similar dispersion for $\mathrm{NO}$. For $\mathrm{SO}_{2}$, the situation is similar although the effect is somewhat smaller for O-rich abundances than for the case of NO. The two main formation reactions for $\mathrm{SO}_{2}$ are the reactions between the radical $\mathrm{SO}$ and $\mathrm{O}$ or $\mathrm{OH}$; both of these reactant partners are reactants in reaction (1).

\subsection{High Sulfur Calculations}

Although chemical models of cold dense clouds often use a low abundance of sulfur to improve the overall agreement with observation, there is little or no evidence of any depletion of this element in diffuse clouds. It thus behooves us to investigate the effects of an abundance of sulfur 2 orders of magnitude greater than listed in Table 1, a value close to what is obtained in diffuse clouds. In Figures 7 and 8 the comparisons of the calculated $\mathrm{O}_{2}$ abundances versus time for models with high and low sulfur are depicted; Figure 7 shows the O-rich case, while Figure 8 shows the $\mathrm{C}$-rich case. In both cases, use of the higher sulfur abundance reduces the calculated $\mathrm{O}_{2}$ abundance. In the O-rich case this effect starts at $\approx 10^{3} \mathrm{yr}$ and increases as time evolves. The largest effect, which is about 1 order of magnitude, occurs at late times, when the lowest $k_{1}$ value is used. Nevertheless, the use of the high sulfur abundance only increases the time at which the calculated $\mathrm{O}_{2}$ abundance surpasses the observed upper limit by a factor of a few. In the C-rich case, the effect is more significant: starting from $\approx 10^{3} \mathrm{yr}$, the effect can be as large as 4 orders of magnitude. Moreover, for all values of the rate coefficient $k_{1}$ utilized, the calculated $\mathrm{O}_{2}$ abundance now lies below the upper limit for all times. We should also mention that use of the $\mathrm{C}$-rich abundances reduces the calculated $\mathrm{H}_{2} \mathrm{O}$ abundance by a significant amount at late times; the large early-time value is not changed much. As discussed above, current sensitivity analyses indicate that calculated and observed upper limits for $\mathrm{H}_{2} \mathrm{O}$ are not in serious disagreement anyway for TMC-1.

The strong improvement for $\mathrm{O}_{2}$ and more moderate improvement for $\mathrm{H}_{2} \mathrm{O}$ when compared with observation for the $\mathrm{C}$-rich case with high sulfur raise two questions: (1) what happens to the 

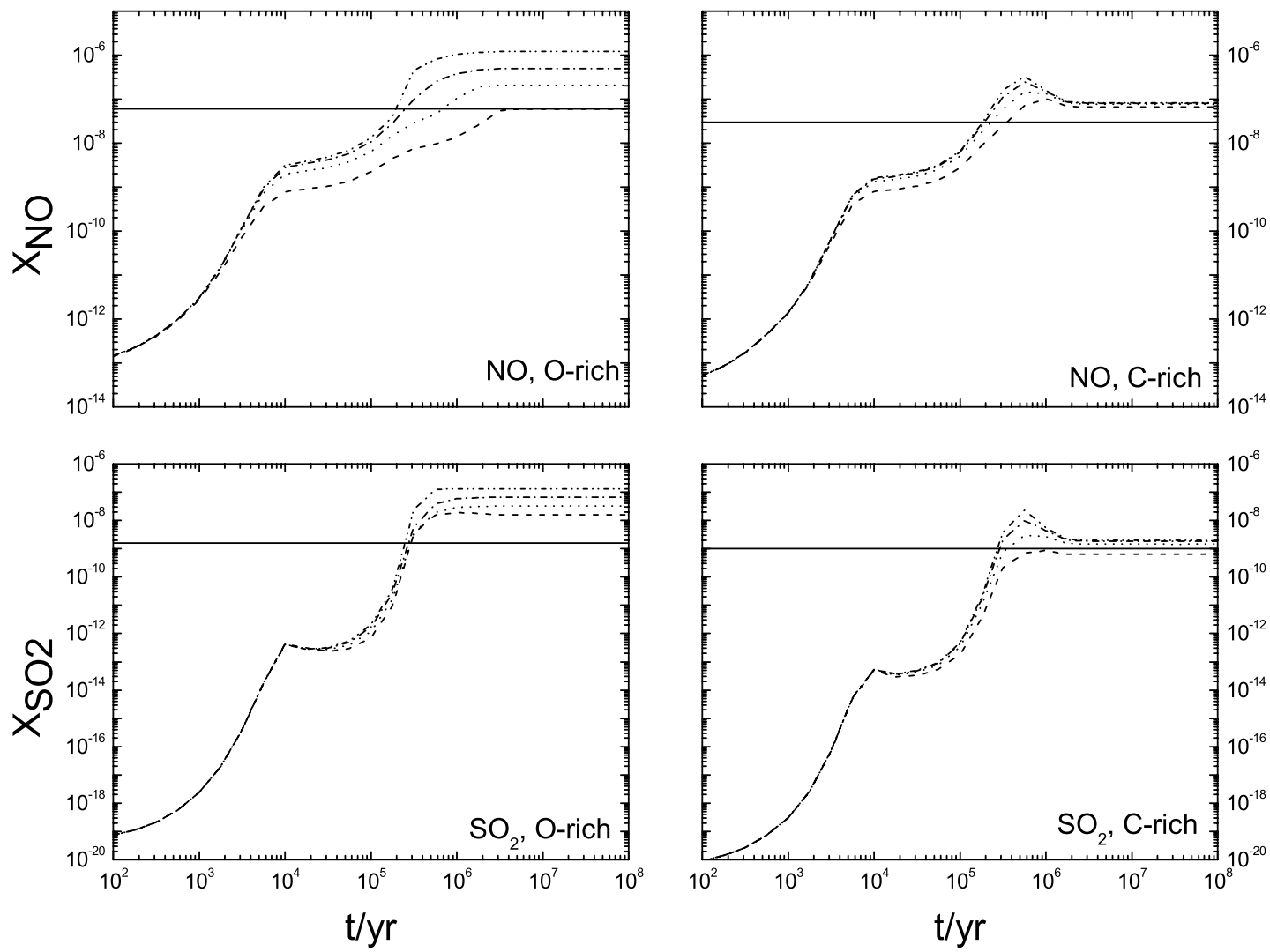

FIg. 6.-Fractional abundances of $\mathrm{NO}$ and $\mathrm{SO}_{2}$ with respect to $\mathrm{H}_{2}$ plotted against time for four different values of the rate coefficient $k_{1}$. The observed fractional abundances or upper limits (Ohishi et al. 1992; Smith et al. 2004) are depicted as solid horizontal lines. C-rich abundances pertain to TMC-1 and O-rich abundances to L134N. See Fig. 1 legend for $k_{1}$ values of different curves.
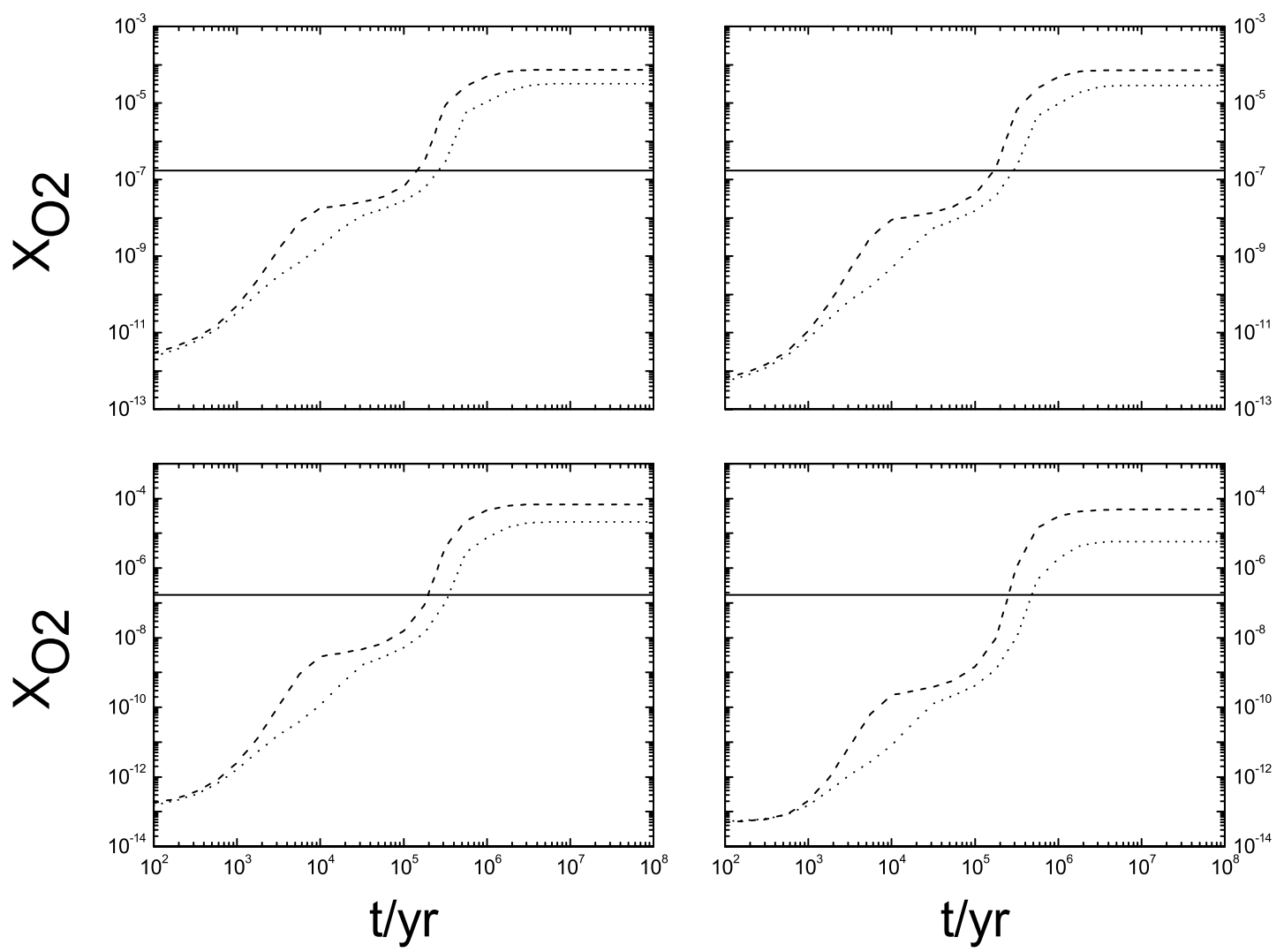

FIG. 7.-Fractional abundance of $\mathrm{O}_{2}$ with respect to $\mathrm{H}_{2}$ plotted against time for four different values of the rate coefficient $k_{1}$. Horizontal solid lines are observed value toward L134N, dashed lines are "low-metal" $\left(\mathrm{O}\right.$-rich) model results, while dotted lines are high-sulfur-model results. Upper left panel: $k_{1}=1.76 \times 10^{-10} \mathrm{~cm}^{3} \mathrm{~s}^{-1} ;$ upper right panel: $k_{1}=3.5 \times 10^{-11} \mathrm{~cm}^{3} \mathrm{~s}^{-1}$; lower left panel: $k_{1}=7.84 \times 10^{-12} \mathrm{~cm}^{3} \mathrm{~s}^{-1}$; lower right panel: $k_{1}=5.4 \times 10^{-13} \mathrm{~cm}^{3} \mathrm{~s}^{-1}$. 

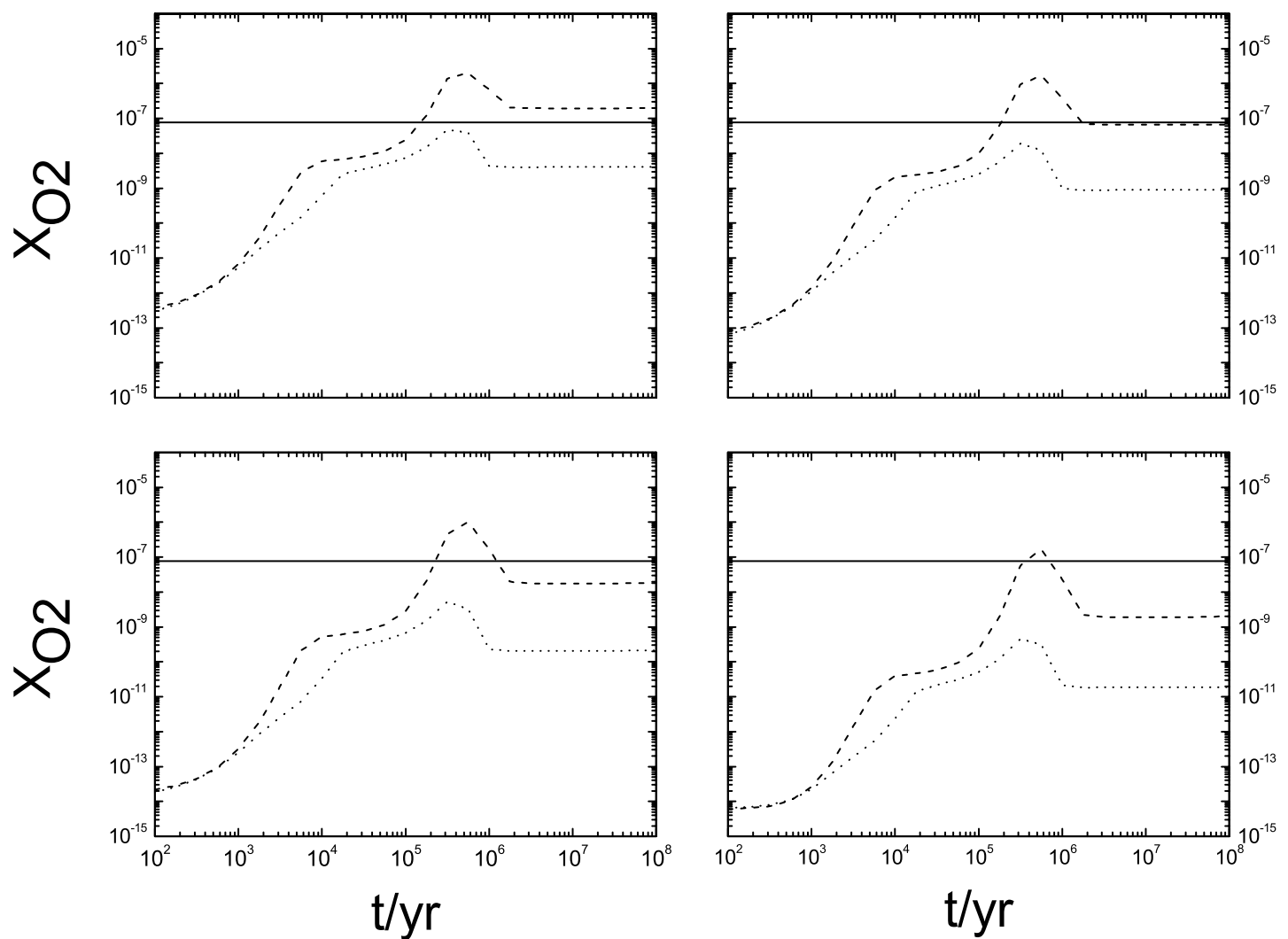

FIG. 8. - Same as Fig. 7, but horizontal solid lines are observed value toward TMC-1, dashed lines are "low-metal" (C-rich) model results, dotted lines are highsulfur-model results.

$\mathrm{OH}$ abundance in this case, and (2) is the general agreement for all molecules as poor as earlier calculations determined? Regarding the first question, the $\mathrm{OH}$ abundance decreases sharply, by an order of magnitude or more, resulting in worse agreement with observation for all times, even remaining slightly outside the order-of-magnitude uncertainty at late times. This reduction is far stronger than in the O-rich case and leads to no late-time dependence at all on $k_{1}$. As for the second question, the overall agreement in TMC-1 is worsened dramatically; with the orderof-magnitude criterion, the agreement at early time goes from $\approx 80 \%$ of the observed molecules to $\approx 60 \%$. On the other hand, the overall agreement in L134N, for which O-rich abundances are used, remains about the same. So, it would seem that the use of a high sulfur abundance is no panacea, especially in the C-rich case. Nor, does the use of a high sulfur abundance help the problem of the water abundance in any dramatic fashion.

\subsection{Other Sources and Oxygen}

Cold dark cores, such as TMC-1 and L134N, are not the only cloud cores searched unsuccessfully for molecular oxygen. In fact, Goldsmith et al. (2000) reported a large number of negative results with the $S W A S$ satellite toward a variety of warmer cores such as OMC-1. Toward one of these cores, $\rho$ Oph A, a wellknown star-forming region, later observations with the Odin satellite revealed a tentative detection of $\mathrm{O}_{2}$ with a fractional abundance of $5 \times 10^{-8}$ relative to $\mathrm{H}_{2}$ (Larsson et al. 2007). In this section we discuss $\mathrm{O}_{2}$ results for warm cores using two types of models: (1) a homogeneous gas-phase model similar to that discussed here for cold cores, but with physical conditions $n=10^{6} \mathrm{~cm}^{-3}$ and $T=40 \mathrm{~K}$ and the use of the $39 \mathrm{~K}$ experimental value for the rate coefficient of the $\mathrm{O}+\mathrm{OH}$ reaction, and (2) a gas-grain warm-up model at the same density similar to that recently proposed by us for L1527 (Hassel et al. 2008) in which warm-up occurs from $10 \mathrm{~K}$ to an asymptotic temperature of $30 \mathrm{~K}$ as a nearby star turns on. Before warm-up, the chemistry evolves at a fixed density of $10^{6} \mathrm{~cm}^{-3}$ for $10^{5} \mathrm{yr}$, during which time heavy depletions of most molecules onto grains occur. During the subsequent warm-up period, desorption occurs at different temperatures for different mantle species; $\mathrm{O}_{2}$ is among the more volatile species and so comes off at relatively low temperatures in the range $20-30 \mathrm{~K}$.

The results of the constant-temperature warm gas-phase models for $\mathrm{O}_{2}$ are not very distinguishable from the low-temperature models with a $k_{1}$ value of $3.5 \times 10^{-11} \mathrm{~cm}^{3} \mathrm{~s}^{-1}$. The results of the warm-up model are shown in Figure 9, where it can be seen that the fractional abundance for gas-phase $\mathrm{O}_{2}$ stays below $10^{-8}$ as

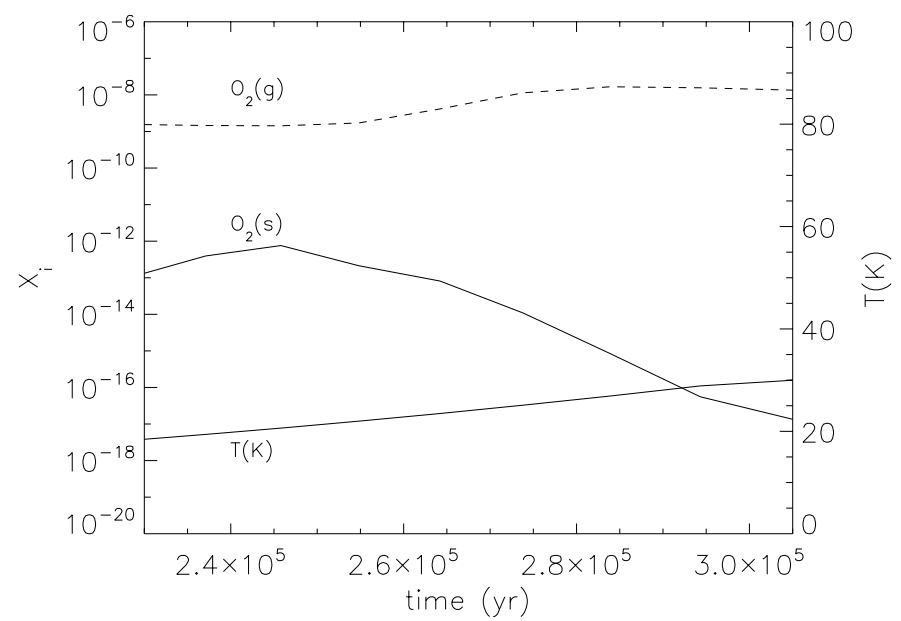

FIG. 9.- Fractional abundance of $\mathrm{O}_{2}$ with respect to $n_{\mathrm{H}}$ plotted against time for warm-up model. See text. 
the warm-up reaches $30 \mathrm{~K}$. This result is in reasonable agreement with both the upper limits detected by SWAS (Goldsmith et al. $2000)$ and the tentative detection of Larsson et al. (2007) in $\rho$ Oph A.

\section{DISCUSSION}

The major purpose of this paper is to explore the effect of changes in $k_{1}$ on the abundance of $\mathrm{O}_{2}$ in cold sources. New calculations of the rate coefficient for reaction (1) show that $k_{1}$ decreases with decreasing temperature from its value at $39 \mathrm{~K}$, which is the lowest temperature explored in the experimental treatment of this reaction (Carty et al. 2006). The value at $10 \mathrm{~K}$, which pertains to cold interstellar cores such as L134N and TMC-1, depends strongly, however, on whether or not an approximation known as the $J$-shifting technique is utilized. Without this approximation, the calculated rate coefficient at $10 \mathrm{~K}$ is only a factor of $\approx 4.5$ times lower than the experimental value at $39 \mathrm{~K}$, but with this approximation, the rate coefficient decreases to a value $\approx 65$ times lower than experiment. Using pseudo-time-dependent model calculations, we have studied the effect of changing the rate coefficient $k_{1}$ on the level of agreement between observed and calculated fractional abundances of $\mathrm{O}_{2}$, its precursor $\mathrm{OH}$, and, to a lesser extent, other affected species in the two cold cores.

Since only an upper limit for $\mathrm{O}_{2}$ can be determined, we require that the theoretical abundance be approximately at or below this limit. With this criterion, the situation for L134N, which is modeled with O-rich elemental abundances (see Figs. 2 and 7), is that all values of $k_{1}$ utilized lead to good agreement with observation for times through $\approx(1-2) \times 10^{5}$ yr only and reach poor agreement shortly thereafter. In other words, the situation regarding $\mathrm{O}_{2}$ for O-rich abundances discussed by Bergin et al. (2000) has not been changed appreciably by variation of $k_{1}$. Nor does the use of a high elemental abundance of sulfur change the situation substantially. The case of TMC-1, which is modeled with C-rich elemental abundances (see Fig. 4), is different once again; here the highest value of $k_{1}$ leads to an $\mathrm{O}_{2}$ abundance that exceeds the upper bound for all times exceeding $2 \times 10^{5} \mathrm{yr}$, as in the O-rich case, but the three lower values of $k_{1}$ lead to $\mathrm{O}_{2}$ abundances that are significantly above the observed upper limit for smaller periods of time. When the high sulfur abundance calculation is pursued, as plotted in Figure 8, all four $k_{1}$ values lead to good agreement with observation at all times. At present, a constraint on the value of $k_{1}$, although it is hardly strong, can be obtained by comparison of calculated and observed $\mathrm{OH}$ abundances in both sources over ranges of time for which the $\mathrm{O}_{2}$ agreement is reasonable. This analysis leads to the superiority of the experimental $\left(3.5 \times 10^{-11} \mathrm{~cm}^{3} \mathrm{~s}^{-1}\right)$ and high-order theoretical values for $k_{1}\left(7.84 \times 10^{-12} \mathrm{~cm}^{3} \mathrm{~s}^{-1}\right)$ over the more extreme upper and lower values, although the $\mathrm{OH}$ observations must be treated with caution.

We have also run some models with the extraordinarily small rate coefficient of $k_{1}=1.0 \times 10^{-15} \mathrm{~cm}^{3} \mathrm{~s}^{-1}$. In this case, for O-rich conditions, the amount of $\mathrm{O}_{2}$ produced at late times is significantly less, and it is produced mainly via other processes. Nevertheless, the calculated fractional abundance of $\approx 10^{-5}$ is still well above the observed upper limit in L134N. For C-rich conditions, on the other hand, the calculated $\mathrm{O}_{2}$ fractional abundance lies below the observed TMC-1 upper limit at all times.

Dust grains also play a role in the gas phase of cold interstellar cores either as passive sites for accretion or sites for diffusive surface reactions followed possibly by nonthermal desorption. Models including such processes tend to lower the abundance of gaseous
$\mathrm{O}_{2}$ at times $\left(\geq 10^{6} \mathrm{yr}\right)$ long enough for sufficient accretion to take place, although surface $\mathrm{O}_{2}$ can be formed slowly by sticking of $\mathrm{O}$ atoms on grain surfaces (Roberts \& Herbst 2002; Garrod et al. 2007). It is not likely that the value of $k_{1}$ in the range considered here will have a major effect at these late times, although its role should be explored.

Let us now assume that in the near future, observations with improved telescopes will result in a detection or reduced upper limits for $\mathrm{O}_{2}$ in cold dense sources. What will the meaning of such observational results be? Our answer must be rather speculative, since our calculated abundance for $\mathrm{O}_{2}$ depends on a variety of parameters and constraints such as the correct elemental abundances, the value of $k_{1}$, and whether or not a purely gas-phase model is used.

Consider a detection or upper limit of an $\mathrm{O}_{2}$ fractional abundance in the vicinity of $10^{-9}$ with a factor of 3 uncertainty, an abundance considerably lower than the current upper limits. For the case of oxygen-rich elemental abundances and the three highest values for $k_{1}$, such a low abundance would require a very young age for the cloud, less than $10^{4} \mathrm{yr}$ for the low sulfur case and perhaps a factor of 3 older for the high sulfur case. Such a young age is incompatible with the observed $\mathrm{OH}$ abundance, even if the uncertainty is large, and moreover worsens the general agreement for all molecules detected in L134N. Indeed, the use of such a young age in a model with uncertain initial conditions and abundances is probably unrealistic and suggests that models in which chemistry occurs as the dense core actually forms be considered. For the lowest value of $k_{1}$, the cloud could be as old as $10^{5}$ or slightly more. At this age the calculated $\mathrm{OH}$ abundance is barely within an order of magnitude of the observed value, but the general agreement for $\mathrm{L} 134 \mathrm{~N}$ is good (Wakelam et al. 2006). On the other hand, the very low value for $k_{1}$ derives from an approximate theory and is unlikely to be accurate.

With carbon-rich abundances, a young cloud age would also be indicated for the low sulfur case, but for the high sulfur case, ages up to $10^{5} \mathrm{yr}$ and even late-time results would indeed be compatible with observation no matter which of our four values of $k_{1}$ is used. The calculated $\mathrm{OH}$ abundance lies within an order of magnitude of the observed value at these times. Of course, the highcarbon high sulfur abundances do not lead to very good general agreement with the more than 50 molecules in TMC-1 at any time. So, we can only suggest that for all the pseudo-time-dependent models considered here, there would be no truly satisfactory explanation for such a low abundance of molecular oxygen, to the best of our knowledge. Even for gas-grain models with accretion and nonthermal desorption, there is great difficulty in reaching such a low gas-phase abundance (Garrod et al. 2007). We are currently exploring models in which the chemistry occurs during the formation of cold dense cores via shock processes.

E. H. acknowledges support for his research program in astrochemistry by the National Science Foundation through grant AST 07-02876 and by NASA grants RSA 1323053 (Jet Propulsion Laboratory) in support of the Herschel telescope and 1290734 (Jet Propulsion Laboratory) in support of the Spitzer telescope. Astrophysics at QUB is supported by a grant from STFC. S. Y. L. and H. G. were supported by the Department of Energy. P. H. thanks the Institut du Développement et des Ressources en Informatique Scientifique (IDRIS, CNRS) for providing the computer power for dynamical calculations. D. X. acknowledges support from the National Natural Science Foundation of China. 
Bergin, E. A., \& Snell, R. L. 2002, ApJ, 581, L105

Bergin, E. A., et al. 2000, ApJ, 539, L129

Carty, D., Goddard, A., Köler, S. P. K., Sims, I. R., \& Smith, I. W. M. 2006, J. Phys. Chem. A, 110, 33101

Charnley, S. B., Rodgers, S. D., \& Ehrenfreund, P. 2001, A\&A, 378, 1024

Davidsson, J., \& Stenholm, L. G. 1990, A\&A, 230, 504

Garrod, R. T., Wakelam, V., \& Herbst, E. 2007, A\&A, 467, 1103

Goldsmith, P. F., et al. 2000, ApJ, 539, L123

Graff, M. M., \& Wagner, A. F. 1990, J. Chem. Phys., 92, 2423

Harding, L. B., Maergoiz, A. I., Troe, J., \& Ushakov, V. G. 2000, J. Chem. Phys., 113, 11019

Harju, J., Winnberg, A., \& Wouterloot, J. G. A. 2000, A\&A, 353, 1065

Hassel, G. E., Herbst, E., \& Garrod, R. T. 2008, ApJ, in press (arXiv0803.1805)

Larsson, B., et al. 2007, A\&A, 466, 999

Le Teuff, Y. H., Millar, T. J., \& Markwick, A. J. 2000, A\&AS, 146, 157

Lin, S. Y., \& Guo, H. 2004, J. Phys. Chem. A, 108, 2141

Lin, S. Y., Guo, H., Honvault, P., \& Xie, D. 2006a, J. Phys. Chem. B, 110, 23641

Lin, S. Y., Guo, H., Honvault, P., Xu, C., \& Xie, D. 2008a, J. Chem. Phys., 128, 014303

Lin, S. Y., Sun, Z., Guo, H., Zhang, D. H., Honvault, P., Xie, D., \& Lee, S.-Y. 2008b, J. Phys. Chem. A, 112, 602

\section{REFERENCES}

Lin, S. Y., Xie, D., \& Guo, H. 2006b, J. Chem. Phys., 125, 091103 Miller, J. A. 1986, J. Chem. Phys., 84, 6170

Ohishi, M., Irvine, W. M., \& Kaifu, N. 1992, in IAU Symp. 150, Astrochemistry of Cosmic Phenomena, ed. P. D. Singh (Dordrecht: Kluwer), 171

Pagani, L., Bacmann, A., Cabrit, S., \& Vastel, C. 2007, A\&A, 467, 179

Pagani, L., et al. 2003, A\&A, 402, L77

Roberts, H., \& Herbst, E. 2002, A\&A, 395, 233

Robertson, R., \& Smith, G. P. 2006, J. Phys. Chem. A, 110, 6673

Smith, I. W. M., Herbst, E., \& Chang, Q. 2004, MNRAS, 350, 323

Smith, I. W. M., \& Stewart, D. W. A. 1994, J. Chem. Soc. Faraday Trans., 90, 3221

Spaans, M., \& van Dishoeck, E. F. 2001, ApJ, 548, L217

Viti, S., Roueff, E., Hartquist, T. W., Pineau des Forêts, G., \& Williams, D. A. 2001, A\&A, 370, 557

Wakelam, V., Herbst, E., \& Selsis, F. 2006, A\&A, 451, 551

Willacy, K., Langer, W. D., \& Allen, M. 2002, ApJ, 573, L119

Xu, C., Jiang, B., Xie, D., Farantos, S. C., Lin, S. Y., \& Guo, H. 2007a, J. Phys. Chem. A, 111, 10353

Xu, C., Xie, D., Honvault, P., Lin, S. Y., \& Guo, H. 2007b, J. Chem. Phys., 127,024304

Xu, C., Xie, D., Zhang, D. H., Lin, S. Y., \& Guo, H. 2005, J. Chem. Phys., 122, 244305 